\title{
Emerging Technology: Preparing Tomorrow's MCH Workforce to Innovate for Equity
}

\author{
Marissa McKool ${ }^{1} \cdot$ Sarah Han ${ }^{1} \cdot$ Jaspal Sandhu ${ }^{1} \cdot$ Cassondra Marshall $^{1} \cdot$ Sylvia Guendelman $^{1} \cdot$ Kim Harley $^{1}$
}

Accepted: 20 December 2021 / Published online: 20 January 2022

(c) The Author(s) 2022

\begin{abstract}
Purpose This commentary proposes a new direction to train the MCH workforce by leveraging today's rapidly changing innovation and technology to address persistent health inequities.

Description We outline the creation of an $\mathrm{MCH}$ technology and innovation training pipeline developed by harnessing creative funding opportunities, diversifying training modalities, and expanding partnerships beyond traditional academic-practice partners, that be replicated and adapted by other academic programs.

Assessment Technology and innovation will continue to be a growing intersection between health and equity, and we must create a robust pipeline of $\mathrm{MCH}$ leaders prepared to collaborate with entrepreneurial and innovation leaders.

Conclusion Technology offers an important opportunity to improve MCH outcomes and reduce disparities, but only if we train the $\mathrm{MCH}$ workforce to seize these opportunities.
\end{abstract}

Keywords $\mathrm{MCH} \cdot$ Technology $\cdot$ Innovation $\cdot$ Workforce development $\cdot$ Training pipeline

\section{Significance Statement}

The landscape of public health and healthcare is rapidly evolving, yet the U.S. continues to face racial disparities in maternal and infant health. The adoption of technology in our daily lives has grown exponentially, especially as a result of the COVID-19 pandemic. Research shows that technology can help reduce barriers to healthcare and provide key insights into health information. However, a critical understanding of how to leverage today's fast growing technology into a new digital age for public health is lacking from current $\mathrm{MCH}$ training. This commentary proposes a new method of developing a training pipeline of leaders in $\mathrm{MCH}$ technology and innovation to address health equity.

Marissa McKool

marissa.mckool@gmail.com

1 Wallace Center for MCAH, UC Berkeley School of Public Health, 2121 Berkeley Way, Berkeley, CA, USA

\section{Introduction}

The public health landscape is rapidly evolving as we reckon with deepening social inequities, burgeoning digital technologies, and the global COVID-19 pandemic. Despite important gains, the U.S. continues to face persistent racial disparities in maternal and infant health that have repercussions across the lifespan for individuals, families, health systems, and communities (Holdt Somer et al., 2017; Howell, 2018; $\mathrm{Lu}, 2018$ ). Meanwhile, adoption of technology in healthcare and research has grown exponentially, through the development of health informatics and electronic health records (EHRs) that allow for improved efficiency and opportunities for data sharing, implementation of telehealth modalities that promote communication between health care providers and patients, search engines (e.g., Google) and social media that make health information more widely accessible, and increased use of smartphones that allow for connectivity between health care consumers and providers (Barclay et al., 2014; McNabb et al., 2006). The use of healthcare technology, such as telehealth and remote patient monitoring, has further expanded during the COVID-19 pandemic in part because of updates in telehealth regulations (Webster, 2020). The pandemic also revealed major gaps in the maternal-child safety net and exposed how years of community 
disinvestment and systemic racism have exacerbated health inequities (McCloskey et al., 2021).

As we examine the skills that the emerging workforce will need, a critical understanding of technology and innovation and their growing role in public health-as well as competence in applying emerging digital strategies-are vital for future MCH practitioners, researchers, and advocates (Czabanowska \& Kuhlmann, 2021; Lu, 2019; Shah et al., 2020; Streeter, 2015). The MCH workforce of tomorrow needs to be equipped to leverage this new digital landscape to address persistent health issues and unlock opportunities to achieve $\mathrm{MCH}$ equity. In this paper, we highlight how the Wallace Center for Maternal, Child, and Adolescent Health at UC Berkeley is engaging and training students at the nexus of technology, innovation, and $\mathrm{MCH}$ to be the next generation of public health leaders.

\section{The Wallace Center for Maternal, Child, and Adolescent Health}

The Wallace Center for Maternal, Child, and Adolescent Health (MCAH) is a multidisciplinary research and training center established in 2015 with an endowment from Helen Wallace, the first Chair of the $\mathrm{MCH}$ program at UC Berkeley's School of Public Health. In the formative development of the center, one of the authors (S.G.) consulted with MCH leaders, faculty, and other key stakeholders across the country to identify current research and training gaps. Stakeholders agreed that tremendous opportunities exist to understand the value and effectiveness of various technologies directed at diverse $\mathrm{MCAH}$ populations. This would require engaging the MCAH communities and populations to continuously assess their interest and actual use of technologies, their preferences, satisfaction and solutions to problems that these technologies present.

Our Center's mission is to conduct actionable research in innovation and technology to advance $\mathrm{MCH}$ and reduce inequities, while engaging community and training future leaders. In 2020, we created a new training pipeline approach in $\mathrm{MCH}$ technology and innovation that focused on three defined areas of technology and innovation-(1) Integrating technology into services; (2) Using technology to gain new research insights; and (3) Redefining innovation in public health.

\section{Creating a Training Pipeline in $\mathrm{MCH}$ Technology and Innovation}

To train future public health leaders in these three specific areas of $\mathrm{MCH}$ technology and innovation, we expanded our interdisciplinary partnership approach, developed creative funding mechanisms, and placed trainees on projects with cutting-edge research methodologies. We intentionally included our Wallace Center faculty members in our training pipeline, all of whom are public health faculty in community health science, supporting them as they expand their own expertise to become principal investigators on digital research initiatives and instructors of new and innovative courses within their topic areas of expertise. Undergraduate, masters, doctoral, and post-doctoral students in public health programs, or technology-focused disciplines (e.g., engineering) with a strong public health interest, obtained new knowledge and skills through innovative courses, research positions, internships, dissertation awards, and fellowships. We strategically designed our training opportunities to include students and faculty from both public health and technology-focused disciplines (e.g., engineering) to foster cross-sector education in $\mathrm{MCH}$.

We leveraged existing partnerships and started new collaborations with diverse stakeholders across technology, healthcare, innovation, and public health. To develop new partnerships, we leveraged the strong relationships of our Dean with technology and healthcare leaders, utilized our expansive alumni base to identify unique project opportunities, and connected with internship host sites to explore collaboration possibilities. Partnerships also included research and teaching collaborations with campus faculty outside of public health in technology-focused departments (e.g., data science).

Table 1 Wallace investments in research and training, May 2020-August 2021

\begin{tabular}{lllcl}
\hline Funding model & Core activity & Wallace investment range & $\begin{array}{l}\text { No. of invest- } \\
\text { ments }\end{array}$ & Catalyzed funding \\
\hline Seed funding & Applied research & $\$ 5000-\$ 50,000$ & 5 & Ongoing external research grants \\
Internship matching & Field training & $\$ 5000-\$ 10,000$ & 11 & Matched funding from 6 host organizations ${ }^{\mathrm{a}}$ \\
External grants & Academic training & $\$ 14,000$ & 1 & Funding from campus grant \\
\hline
\end{tabular}

${ }^{a}$ Three host organizations had more than one student placement. Placements at two community-based organizations were fully funded by the Wallace Center due to the organization's limited financial resources 
We used three creative funding mechanisms to provide training opportunities (Table 1). First, we invested seed funding from our center's endowment to support student research positions on five Wallace faculty-led pilot projects. The funding support varied by project need and ranged from $\$ 5000$ to $\$ 50,000$ per project. This seed funding functioned as a grant and differed from departmental funding as they incentivize faculty to incorporate innovation and technology into their research. Second, we created a summer internship placement program for Master of Public Health (MPH) students through a matched funding model. The Wallace Center and a host organization co-funded a student position to work on a technology or innovation-based project, resulting in more student internship opportunities. In the past two years, eleven MPH students completed their internship at eight different organizations ranging from private consulting firms to healthcare providers. Students were paid $\$ 10,000$ for their three-month, full-time placement. Third, our faculty members secured a training grant from our campus to create and implement an applied research undergraduate course in $\mathrm{MCH}$ infodemiology, a research method to assess online search-term information to inform public health (Guendelman et al., 2020; Zepecki et al., 2020). This grant provided funding for a year-long course and one graduate student instructor. To increase support for the applied training, the Wallace Center provided funding for an additional student instructor. The purpose of these projects are to advance $\mathrm{MCH}$ research and training opportunities, with the potential to secure additional external research grants.

Below, we describe the three defined areas of technology and innovation and highlight a specific training opportunity provided by the Wallace Center for each area (see Table 2 . for an expanded list of examples). The Wallace Center takes a life course approach to $\mathrm{MCH}$ research and the projects described here focus on maternal, reproductive health, and family planning.

\section{Integrating Technology into Services}

An expanded integration of technology in care has the potential to address the social determinants of health contributing to health disparities by improving access and reducing barriers to healthcare and other wrap around services. Telehealth services can help extend healthcare to rural areas and underserved urban communities by reducing challenges around transportation, childcare, and work schedules (Hoffman, 2020; Meyer et al., 2020). The use of remote monitoring tools, such as wearable health devices or mobile tracking apps, can improve at-home management of chronic health conditions through the collection of real-time data (Fischer \& Kleen, 2021; Milne-Ives et al., 2020; Vaghefi \& Tulu, 2019).
We advised on the design of a text-based blood pressure monitoring intervention for patients with hypertensive disorders of pregnancy for a county hospital in Oakland, CA delivering nearly 1000 births in California's Medicaid program per year. A UC Berkeley MPH student served as a graduate student researcher on the project in Spring 2021. By conducting the initial literature review for the project and working with clinicians, the student developed unique expertise in telehealth and remote patient monitoring (RPM) research evidence that otherwise would not have been obtained in their coursework. The OB/GYN provider on the project and Wallace faculty advisor (K.H.) also expanded their understanding and knowledge of perinatal telehealth and RPM by learning from the student. In addition, the student worked closely with the OB/GYN provider to design the study and learned about the challenges, opportunities, and complexities of implementing innovative and technology-based interventions in publicly funded healthcare systems. The student has since graduated and secured a fulltime position conducting academic research on digital health and health systems.

\section{Using Technology to Gain New Insights}

Traditional epidemiologic research relies on enrollment, surveying, and follow-up of individual study participants, which can be resource heavy. Millions of consumers now provide real-time quantitative health data, also known as "big data," into mobile apps and other devices that can be longitudinal, quickly synchronized, and are less likely to suffer from recall bias, social desirability bias, or mistakes occurring during manual data transfers (Fischer \& Kleen, 2021). Big data also encompass qualitative data from social media sites such as Reddit, Facebook, and Twitter that can be used to conduct digital ethnographies or infodemiology analyses about public health issues (Eysenbach, 2009; von Hippel, 2018).

One of the authors (C.M.) is a family planning researcher and is assessing the value of social media and online community data for contraception research by conducting a qualitative analysis of a birth control Reddit community. This work utilizes a novel machine learning-enabled approach to analyze user-generated big data from digital communication platforms (von Hippel, 2018). In Spring 2021, a doctoral student from the School of Social Welfare with a designated emphasis in computational data science and engineering joined the research team. The student's expertise in data science enabled the faculty to gain a deeper understanding of the use and limitations of machine learning methodologies for $\mathrm{MCH}$ research. Machine learning describes a set of techniques that run analytical models by adapting to data patterns (Mooney \& Pejaver, 2018). By conducting the analysis of user-generated comments, the student gained a deep understanding of family planning issues, such as lack of 


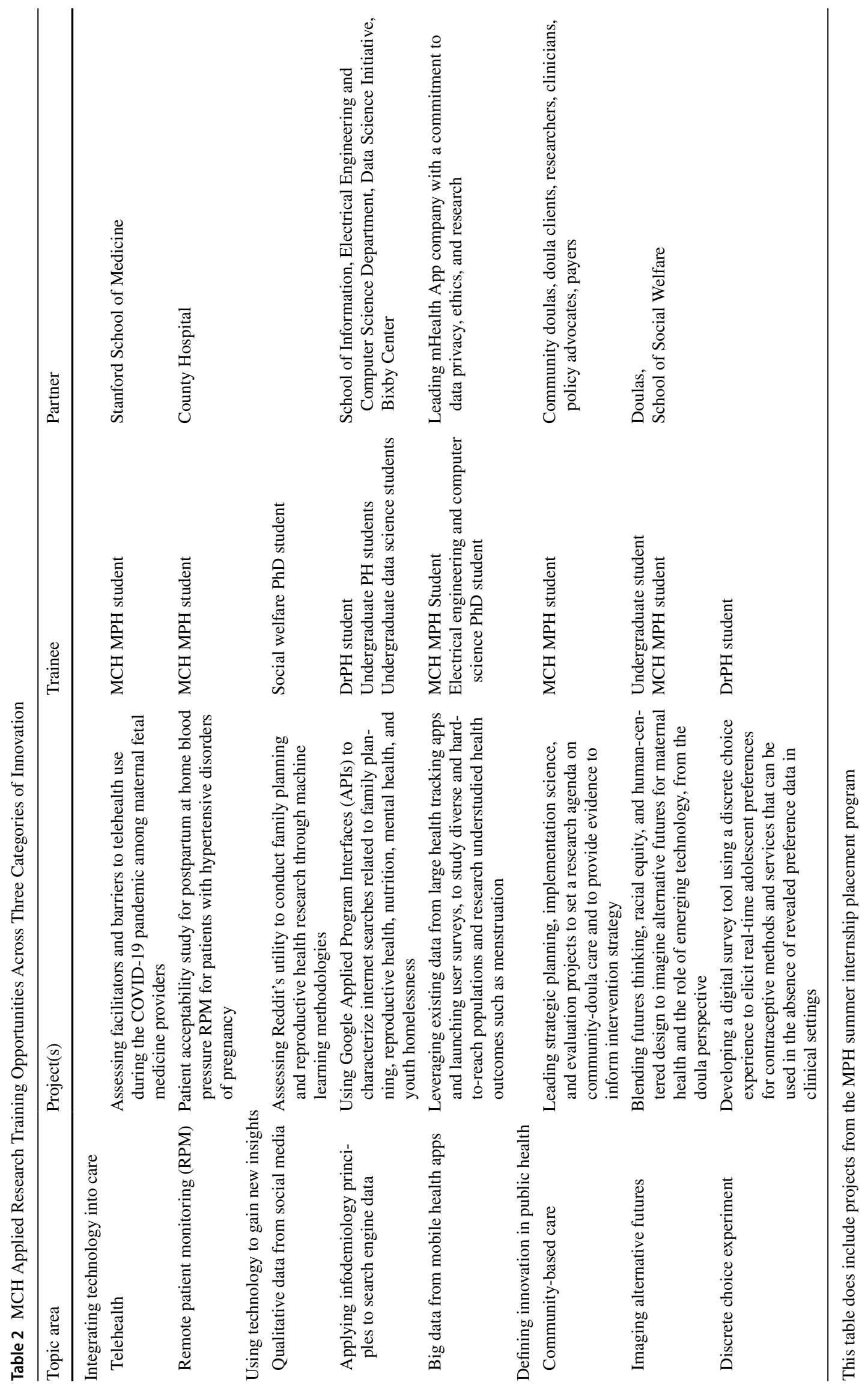


access to contraceptives, experienced by Reddit users. The student also received education and mentorship on $\mathrm{MCH}$ equity issues and public health research ethics that likely would not have been gained in their program coursework or other research opportunities. As a result of this project, the student is now working on a manuscript outlining their findings and applying other machine learning algorithms to the data.

\section{Defining Innovation in Public Health}

Leveraging human-centered design approaches has strong potential for developing novel interventions to improve MCH (Vechakul et al., 2015). Human-centered design is an innovative strategy that involves first cultivating deep empathy with users and understanding their needs before defining the problem at hand. The next steps are to ideate solutions and then prototype and test them in creative, interactive ways in direct collaboration with the users (Lister et al., 2017). This ensures that the resulting solution or product is engaging, adaptive, and relevant to the specific user group it was designed for.

We are blending futures thinking, racial equity, and human-centered design to imagine alternative futures for maternal health and the role of emerging technology by gathering doula perspectives for the future of maternal health care. The project team consisted of a faculty lead (J.S.), who has a background in engineering and design thinking, and an advisory board of faculty experts in qualitative doula research and practicing community doulas. The research team included an undergraduate public health student, an MCH MPH student, and a Boston University MCH student between Spring and Summer 2021. These students gained insights into human-centered design methodologies, as well as traditional qualitative research. In addition, the advisory board members broadened their understanding of design thinking principles and methodologies from the core research team and the faculty lead deepened his understanding of entrenched issues in MCH equity and maternal health care. In the Fall 2021, students will be holding design thinking sessions with the project partners and doulas.

\section{Opportunities to Innovate MCH Training}

The MCH perspective is a critical but largely unheard voice for the prioritization and development of innovative and technological solutions to entrenched public health problems. The MCH workforce is poised to center equity and engage hard-to-reach and underrepresented populations, outline novel ethical research methodologies, and build a comprehensive evidence-base for interventions (digital and otherwise). If we do not train our $\mathrm{MCH}$ workforce in the space of innovation and technology, we risk losing opportunities in the development and dissemination of cuttingedge research and interventions to address entrenched $\mathrm{MCH}$ issues. Given the rapid evolution of technology, we must be agile in leveraging innovative and digital solutions for public health research and practice to advance health equity. We need trained $\mathrm{MCH}$ professionals at the table, alongside experts and decision-makers in business, medicine, data and computer science, and other technology sectors.

Developing and implementing dynamic, high-quality, engaging, and diverse training opportunities that incorporate technological innovations is feasible for public health with relatively limited investment. The Wallace Center's work described here can be replicated by other academic programs and Title $\mathrm{V}$ programs can partner with these programs. Using creative funding models-such as investing seed funding and matching funds with private partners-can create new training opportunities with relatively lower start-up funds. This requires reimagining academic collaboration with the private sector, including with entrepreneurial partners. It also requires transforming cross-academic partnerships to engage technology-driven departments. Building these new partnerships allows public health to leverage additional resources in partnership with technology and business. Diversifying project teams to include public health and technology perspectives also allows for organic training in $\mathrm{MCH}$ and innovation across trainees. Similarly, diversifying the modality of trainingfrom applied courses to internship placements-expands the opportunities for training, partnership, and impact.

Technology is here to stay and its intersections with health and equity will continue to grow. To ensure future $\mathrm{MCH}$ leaders are prepared to serve at this intersection, we must expand $\mathrm{MCH}$ training to include applied knowledge and skills in technology and innovation so they are prepared to center the needs and voices of underserved populations, leverage novel research methods that are ethical and sound, and develop and evaluate innovative interventions in direct collaboration with a diverse range stakeholders.

Author Contributions MM: Conceptualization, writing-original draft, review and editing, project administration, supervision. $\mathrm{SH}$ : Conceptualization, writing - original draft, review and editing, project administration. JS: Conceptualization, writing-review and editing. CM: Writing — review and editing. SG: Writing — review and editing. $\mathrm{KH}$ : Conceptualization, writing-review and editing.

Funding This work was funded by the endowment of the Wallace Center in MCAH from Helen Wallace, established in 2015.

Data Availability Not applicable.

Code Availability Not applicable. 


\section{Declarations}

Conflict of interest Not applicable.

Ethical Approval Not applicable.

Consent to Participate Not applicable.

Consent for Publication Not applicable.

Open Access This article is licensed under a Creative Commons Attribution 4.0 International License, which permits use, sharing, adaptation, distribution and reproduction in any medium or format, as long as you give appropriate credit to the original author(s) and the source, provide a link to the Creative Commons licence, and indicate if changes were made. The images or other third party material in this article are included in the article's Creative Commons licence, unless indicated otherwise in a credit line to the material. If material is not included in the article's Creative Commons licence and your intended use is not permitted by statutory regulation or exceeds the permitted use, you will need to obtain permission directly from the copyright holder. To view a copy of this licence, visit http://creativecommons.org/licenses/by/4.0/.

\section{References}

Barclay, G., Sabina, A., \& Graham, G. (2014). Population health and technology: Placing people first. American Journal of Public Health, 104(12), 2246-2247. https://doi.org/10.2105/AJPH.2014. 302334

Czabanowska, K., \& Kuhlmann, E. (2021). Public health competences through the lens of the COVID-19 pandemic: What matters for health workforce preparedness for global health emergencies. The International Journal of Health Planning and Management, 36(S1), 14-19. https://doi.org/10.1002/hpm.3131

Eysenbach, G. (2009). Infodemiology and Infoveillance: Framework for an emerging set of public health informatics methods to analyze search, communication and publication behavior on the internet. Journal of Medical Internet Research, 11(1), e1157. https:// doi.org/10.2196/jmir.1157

Fischer, F., \& Kleen, S. (2021). Possibilities, problems, and perspectives of data collection by mobile apps in longitudinal epidemiological studies: Scoping review. Journal of Medical Internet Research, 23(1), e17691. https://doi.org/10.2196/17691

Guendelman, S., Yon, E., Pleasants, E., Hubbard, A., \& Prata, N. (2020). Shining the light on abortion: Drivers of online abortion searches across the United States in 2018. PLoS ONE, 15(5), e0231672. https://doi.org/10.1371/journal.pone.0231672

Hoffman, D. A. (2020). Increasing access to care: Telehealth during COVID-19. Journal of Law and the Biosciences. https://doi.org/ 10.1093/jlb/lsaa043

Holdt Somer, S. J., Sinkey, R. G., \& Bryant, A. S. (2017). Epidemiology of racial/ethnic disparities in severe maternal morbidity and mortality. Seminars in Perinatology, 41(5), 258-265. https://doi. org/10.1053/j.semperi.2017.04.001

Howell, E. A. (2018). Reducing disparities in severe maternal morbidity and mortality. Clinical Obstetrics and Gynecology, 61(2), 387-399. https://doi.org/10.1097/GRF.0000000000000349

Lister, C., Payne, H., Hanson, C. L., Barnes, M. D., Davis, S. F., \& Manwaring, T. (2017). The public health innovation model:
Merging private sector processes with public health strengths. Frontiers in Public Health. https://doi.org/10.3389/fpubh.2017. 00192

Lu, M. C. (2018). Reducing maternal mortality in the United States. JAMA, 320(12), 1237-1238. https://doi.org/10.1001/jama.2018. 11652

Lu, M. C. (2019). The future of maternal and child health. Maternal and Child Health Journal, 23(1), 1-7. https://doi.org/10.1007/ s10995-018-2643-6

McCloskey, L., Amutah-Onukagha, N., Bernstein, J., \& Handler, A. (2021). Setting the agenda for reproductive and maternal health in the era of COVID-19: Lessons from a cruel and radical teacher. Maternal and Child Health Journal, 25(2), 181-191. https://doi. org/10.1007/s10995-020-03033-y

McNabb, S. J. N., Koo, D., Seligman, J., Centers for Disease Control and Prevention. (2006). Informatics and public health at CDC. MMWR Supplements, 55(2), 25-28.

Meyer, C., Becot, F., Burke, R., \& Weichelt, B. (2020). Rural telehealth use during the COVID-19 pandemic: How long-term infrastructure commitment may support rural health care systems resilience. Journal of Agromedicine. https://doi.org/10.1080/1059924X. 2020.1814921

Milne-Ives, M., van Velthoven, M. H., \& Meinert, E. (2020). Mobile apps for real-world evidence in health care. Journal of the American Medical Informatics Association: JAMIA, 27(6), 976-980. https://doi.org/10.1093/jamia/ocaa036

Mooney, S. J., \& Pejaver, V. (2018). Big data in public health: Terminology, machine learning, and privacy. Annual Review of Public Health, 39(1), 95-112. https://doi.org/10.1146/annurev-publh ealth-040617-014208

Shah, G., Bogaert, K., Yin, J., \& Gould, E. (2020). Gaps in public health workforce awareness of emerging public health trends. Journal of Public Health Management and Practice: JPHMP, 26, 401-403. https://doi.org/10.1097/PHH.0000000000001049

Streeter, N. (2015). Workforce crisis in MCH leadership. Maternal and Child Health Journal, 19(2), 240-243. https://doi.org/10.1007/ s10995-014-1621-x

Vaghefi, I., \& Tulu, B. (2019). The continued use of mobile health apps: Insights from a longitudinal study. JMIR MHealth and UHealth. https://doi.org/10.2196/12983

Vechakul, J., Shrimali, B. P., \& Sandhu, J. S. (2015). Human-centered design as an approach for place-based innovation in public health: A case study from Oakland, California. Maternal and Child Health Journal, 19(12), 2552-2559. https://doi.org/10.1007/ s10995-015-1787-x

von Hippel, C. (2018). A next generation assets-based public health intervention development model: The public as innovators. Frontiers in Public Health, 6, 248. https://doi.org/10.3389/fpubh.2018. 00248

Webster, P. (2020). Virtual health care in the era of COVID-19. The Lancet, 395(10231), 1180-1181. https://doi.org/10.1016/S01406736(20)30818-7

Zepecki, A., Guendelman, S., DeNero, J., \& Prata, N. (2020). Using application programming interfaces to access google data for health research: Protocol for a methodological framework. JMIR Research Protocols, 9(7), e16543. https://doi.org/10.2196/16543

Publisher's Note Springer Nature remains neutral with regard to jurisdictional claims in published maps and institutional affiliations. 